\title{
Realities and misconceptions on the pericytes role in tissue repair
}

\author{
Paola Campagnolo1, Rajesh Katare ${ }^{2}$ \& Paolo Madeddu*,3 \\ ${ }^{1}$ Faculty of Health \& Medical Sciences, School of Biosciences \& Medicine, Section of Cardiovascular Sciences, University of Surrey, \\ Guildford GU2 7XH, UK \\ ${ }^{2}$ Department of Physiology, Otago School of Medical Sciences, University of Otago, Dunedin, New Zealand \\ ${ }^{3}$ Bristol Heart Institute, School of Clinical Sciences, University of Bristol, Bristol BS2 8HW, UK \\ * Author for correspondence: Tel.: +44 117342 3904; Fax: +44 117342 3904; mdprm@bristol.ac.uk
}

'For many years, pericytes were not considered to merit scientific or medical attention. A revival of pericytes started between 1960 and 1990, when a series of seminal studies showed that osteogenic, multipotent, fibroblast colony-forming units could be found both in the bone marrow and in the connective tissue of several organs, including the heart"

First draft submitted: 11 June 2017; Accepted for publication: 30 January 2018; Published online: 19 March 2018

Keywords: mesenchymal stem cells $\bullet$ multipotency $\bullet$ pericytes $\bullet$ regeneration

Pericytes were first described by Rouget in 1873 as 'nonpigmented contractile adventitial cells' that encircle capillaries with cytoplasmic processes. For many years, pericytes were not considered to merit scientific or medical attention. A revival of pericytes started between 1960 and 1990, when a series of seminal studies showed that osteogenic, multipotent, fibroblast colony-forming units could be found both in the bone marrow and in the connective tissue of several organs, including the heart [1,2]. Eventually, the in vivo identity of these mesodermal progenitors has been extensively elucidated and now pericytes are attracting much attention as a potential cell candidate for regenerative medicine treatments.

\section{The perivascular niche as a source of stem cells}

Bianco and Cossu were first to propose a perivascular/mural origin for these cells [3]. Importantly, clonogenic assays and cell fate tracking experiments suggested an overlapping between mesodermal and angiogenic progenitor cells, which may share their origin from the mesoangioblast, a cell type that initially identified within the hemogenic endothelium of the embryonic dorsal aorta [4,5]. In addition, osteoprogenitor cells from the bone marrow vascular niche could be prospectively enriched by employing CD146, an antigen shared with pericytes/mural cells [6]. In analogy, a myogenic population, antigenically and anatomically distinct from satellite cells, was identified in skeletal muscles, which express typical pericyte markers (including NG2, alkaline phosphatase and PDGFRb) [7]. These results were later extended by identifying in situ and prospectively sorting cells that possess a pericyte phenotype, coexpress mesenchymal stem cell (MSC) markers, and are endowed of clonogenicity and multipotency properties [8]. However, both the possibilities that pericyte subsets act as bona fide MSCs and that pericytes are a uniform population of cells that can be cultured as MSCs have been questioned. Specifically, studies conducted on mesodermal progenitors isolated from different sources indicate that CD146-expressing perivascular cells are clearly distinct from the bone marrow-derived ones, both from a potency and transcriptional standpoint [9-11]. Furthermore, CD146 is not a universal marker of pericytes, because it is also expressed by endothelial cells but not by CD34-expressing adventitial pericytes, which localize in the external layer of large vessel nearby the vasa vasorum [12]. Intriguingly, increasing experimental evidence indicates that tissue-specific mesodermal progenitors could be recruited to a mural fate, thus suggesting that pericytes might serve as local stem/progenitor cells [11].

\section{Lineage tracing studies suggest pericytes are fake stem cells}

A recent article from Guimarães-Camboa et al. has generated much discussion among vascular biologists and 'pericytologists'. The reason for the sensational impact of this new article is that the authors provide several 
arguments refuting the capacity of pericytes to behave like multipotent tissue-resident progenitors [13]. Indeed, their multipotency as well as that attributed to MSCs might be an in vitro artifact. Using an inducible lineagetracing Tbx18-CreERT2 system, Guimarães-Camboa et al. documented that perivascular cells maintain their identity in situ, during aging and under diverse pathological settings. They also argue against the existence of a hierarchical association between pericytes and MSCs previously proposed by Peault and colleagues, who showed that transplanted perivascular cells contribute to tissue-specific lineages in vivo $[8,14]$. The editorial comment to the Guimarães-Camboa's study pinpoints the urgent need for a better definition and understanding of the pathophysiological role of perivascular MSCs, both in their native niche and as therapeutic tools [15].

\section{Dimensioning the judgment from sensationalism}

The merit of sensational studies is that they 'stir the pot' creating new awareness and dismantling prepackaged dogmas. The demerit is that another dogma is often generated in order to recreate the cohesion essential to a wellfunctioning scientific constitution. In case like this, a deep and honest discussion on available evidence is warranted to contrast the modern attitude of scientists to throw the water and the child. Here, we wish to contribute to the debate by discussing some potential bias of the ground-breaking experimental study by Guimarães-Camboa and contextualizing their results within the framework of recent research on pericytes. First, the study presents some potential technical limitations that need to be acknowledged. Tamoxifen induction requires careful titration to ensure complete recombination in the target tissue [16] and has previously been shown to cause some illegitimate recombination, leading to pathological consequences $[17,18]$. Furthermore, this process has been associated with inflammation, reduced cell proliferation and increased apoptosis in a dose-dependent manner [18-20]. Loss of Tbx18-CreERT2 cells by 'off-target' apoptosis or inflammatory/immune responses might lead to underestimation of perivascular cells-dependent age- or injury-related tissue remodeling.

More crucially, while Tbx18-Cre lineage tracing was previously used to identify the developing epicardial pericytes and coronary vascular smooth muscle cells [21], the role and fate of pericytes that do not express Tbx18 have not been elucidated in Guimarães-Camboa's study. Indeed, an intriguing possibility is that a small fraction of Tbx18negative cells represents the multipotent perivascular population that the authors were hunting for. The vasculature is a slow cellular turnover tissue and hence not abundant in multipotent stem cells. Accordingly, we demonstrated that only approximately $2 \%$ of single-sorted human pericytes grew to form secondary colonies through two subsequent cloning passages [12]. The secondary clones conserved the mesenchymal/pericyte antigenic phenotype and showed a wide differentiation capacity following exposure to inductive stimuli, giving rise to mesodermal lineages and neuron-like cells in vitro. We envision combining clonal and lineage tracing analyses to determine if Tbx18-negative pericytes represent the multipotent progenitors of the lineage-restricted Tbx18-positive pericytes.

\section{Is multipotency really necessary for regeneration?}

An additional comment is appropriate on the implication of the Guimarães-Camboa's study to the disputed field of regenerative medicine. MSC therapy has been already used in the clinic for approximately 10 years [22], and it is now widely accepted that these cells promote tissue healing through paracrine mechanisms rather than by differentiation [23]. Nevertheless, to the best of our knowledge, no clinical trial with pericytes has been conducted so far. Preclinical studies of human pericyte therapy from our group and others showed encouraging results in xenogeneic murine models of peripheral and myocardial ischemia. The underpinning healing mechanisms consist of stimulation of angiogenesis, promotion of myocyte survival and proliferation, and recruitment of resident progenitor cells $[12,14,24,25]$. One step forward to clinical application, we have confirmed transplantation of allogeneic pericytes promotes reparative angiogenesis in a swine model of reperfused myocardial infarction [26]. Moreover, our studies demonstrated that pericytes retain their original antigenic characteristics and a 'perivascular memory' upon transplantation into the mouse infarcted heart, migrating from the injection site to localize in close contact with capillary endothelial cells. This tight juxtaposition allows the transfer of paracrine signals, among the others the proangiogenic miR-132, from transplanted pericytes to resident vascular cells. Extensive histological investigation of human pericytes-transplanted hearts excluded the presence of calcification, frequently observed in MSC-transplanted hearts [24]. Likewise, in agreement with Guimarães-Camboa's study, we could not detect differentiation of transplanted pericytes into myocytes [25]. These properties make pericytes a promisingly safe cell product in comparison with other competitive solutions. It should be pointed out that our studies employed heterogeneous pericyte populations; additional studies are warranted to assess the in vivo differentiation activity and healing potential of pericyte colony-forming units. 


\section{Conclusion \& clinical perspective}

Pericyte dysfunction is a common feature of many vascular pathologies, where loss of mural coverage contributes to retinopathy, nephropathy, myopathies, myocardial edema and postischemic coronary no reflow [27]. An increasing understanding of the molecular mechanisms underpinning pericyte dysfunction is expected to open new avenues to the treatment of a spectrum of microvascular diseases that affect million patients worldwide [28]. This objective could be achieved through several means, including a classical pharmacological approach targeting the molecular deficit of resident pericytes or supply side reconstitution by naive or genetically modified pericytes. Furthermore, pericytes could be employed, alone or in combination with other vascular cells and parenchymal cells, to generate tissue-engineered grafts to replace or repair damaged organs. Therefore, pericytes might not be the progenitors of MSCs, as proposed by Guimarães-Camboa et al., yet they maintain an intact appeal with preclinical evidence supporting a key role in tissue repair and plenty more to come.

\section{Financial \& competing interests disclosure}

The authors have no relevant affiliations or financial involvement with any organization or entity with a financial interest in or financial conflict with the subject matter or materials discussed in the manuscript. This includes employment, consultancies, honoraria, stock ownership or options, expert testimony, grants or patents received or pending, or royalties.

No writing assistance was utilized in the production of this manuscript.

\section{References}

1. Young HE, Mancini ML, Wright RP et al. Mesenchymal stem cells reside within the connective tissues of many organs. Dev. Dyn. 202(2), 137-144 (1995).

2. Friedenstein AJ, Piatetzky S II, Petrakova KV. Osteogenesis in transplants of bone marrow cells. J. Embryol. Exp. Morphol. 16(3), 381-390 (1966).

3. Bianco P, Cossu G. Uno, nessuno e centomila: searching for the identity of mesodermal progenitors. Exp. Cell Res. 251(2), 257-263 (1999).

4. Minasi MG, Riminucci M, De Angelis L et al. The meso-angioblast: a multipotent, self-renewing cell that originates from the dorsal aorta and differentiates into most mesodermal tissues. Development 129(11), 2773-2783 (2002).

5. Azzoni E, Conti V, Campana $\mathrm{L}$ et al. Hemogenic endothelium generates mesoangioblasts that contribute to several mesodermal lineages in vivo. Development 141(9), 1821-1834 (2014).

6. Sacchetti B, Funari A, Michienzi $S$ et al. Self-renewing osteoprogenitors in bone marrow sinusoids can organize a hematopoietic microenvironment. Cell 131(2), 324-336 (2007).

7. Dellavalle A, Sampaolesi M, Tonlorenzi R et al. Pericytes of human skeletal muscle are myogenic precursors distinct from satellite cells. Nat. Cell Biol. 9(3), 255-267 (2007).

8. Crisan M, Yap S, Casteilla L et al. A perivascular origin for mesenchymal stem cells in multiple human organs. Cell stem cell 3(3), 301-313 (2008).

9. Verardo R, Piazza S, Klaric E et al. Specific mesothelial signature marks the heterogeneity of mesenchymal stem cells from high-grade serous ovarian cancer. Stem Cells 32(11), 2998-3011 (2014).

10. Chong JJ, Chandrakanthan V, Xaymardan M et al. Adult cardiac-resident MSC-like stem cells with a proepicardial origin. Cell stem cell 9(6), 527-540 (2011).

11. Sacchetti B, Funari A, Remoli C et al. No identical 'mesenchymal stem cells' at different times and sites: human committed progenitors of distinct origin and differentiation potential are incorporated as adventitial cells in microvessels. Stem cell reports 6(6), 897-913 (2016).

12. Campagnolo P, Cesselli D, Al Haj Zen A et al. Human adult vena saphena contains perivascular progenitor cells endowed with clonogenic and proangiogenic potential. Circulation 121(15), 1735-1745 (2010).

13. Guimarães-Camboa N, Cattaneo P, Sun Y et al. Pericytes of multiple organs do not behave as mesenchymal stem cells in vivo. Cell stem cell 20(3), 345.e5-359.e5 (2017).

14. Chen WC, Baily JE, Corselli M et al. Human myocardial pericytes: multipotent mesodermal precursors exhibiting cardiac specificity. Stem Cells 33(2), 557-573 (2015).

15. Cano E, Gebala V, Gerhardt H. Pericytes or mesenchymal stem cells: is that the question? Cell stem cell 20(3), 296-297 (2017).

16. Reinert RB, Kantz J, Misfeldt AA et al. Tamoxifen-induced Cre-loxP recombination is prolonged in pancreatic islets of adult mice. PLoS One 7(3), e33529 (2012).

17. Harno E, Cottrell EC, White A. Metabolic pitfalls of CNS Cre-based technology. Cell Metab. 18(1), 21-28 (2013).

18. Lexow J, Poggioli T, Sarathchandra P, Santini MP, Rosenthal N. Cardiac fibrosis in mice expressing an inducible myocardial-specific Cre driver. Dis. Model. Mech. 6(6), 1470-1476 (2013). 
19. Bersell K, Choudhury S, Mollova M et al. Moderate and high amounts of tamoxifen in $\alpha$ MHC-MerCreMer mice induce a DNA damage response, leading to heart failure and death. Dis. Model. Mech. 6(6), 1459-1469 (2013).

20. Koitabashi N, Bedja D, Zaiman AL et al. Avoidance of transient cardiomyopathy in cardiomyocyte-targeted tamoxifen-induced MerCreMer gene deletion models. Circ. Res. 105(1), 12-15 (2009).

21. Volz KS, Jacobs AH, Chen HI et al. Pericytes are progenitors for coronary artery smooth muscle. eLife 4 (2015).

22. Karantalis V, Hare JM. Use of mesenchymal stem cells for therapy of cardiac disease. Circ. Res. 116(8), 1413-1430 (2015).

23. Gnecchi M, He H, Liang OD et al. Paracrine action accounts for marked protection of ischemic heart by Akt-modified mesenchymal stem cells. Nat. Med. 11(4), 367-368 (2005).

24. Katare R, Riu F, Mitchell K et al. Transplantation of human pericyte progenitor cells improves the repair of infarcted heart through activation of an angiogenic program involving micro-RNA-132. Circ. Res. 109(8), 894-906 (2011).

25. Avolio E, Meloni M, Spencer HL et al. Combined intramyocardial delivery of human pericytes and cardiac stem cells additively improves the healing of mouse infarcted hearts through stimulation of vascular and muscular repair. Circ. Res. 116(10), E81-E94 (2015).

26. Alvino VV, Fernández-Jiménez R, Rodriguez-Arabaolaza ITransplantation of allogeneic pericytes improves myocardial vascularization and reduces interstitial fibrosis in a swine modelof reperfused acute myocardial infarction. J. Am. Heart Assoc. 7(2), doi:10.1161/JAHA.117.006727 (2018).

27. Avolio E, Madeddu P. Discovering cardiac pericyte biology: from physiopathological mechanisms to potential therapeutic applications in ischemic heart disease. Vascul. Pharmacol. 86, 53-63 (2016).

28. Vono R, Fuoco C, Testa $S$ et al. Activation of the pro-oxidant PKCbetaII-p66Shc signaling pathway contributes to pericyte dysfunction in skeletal muscles of patients with diabetes with critical limb ischemia. Diabetes 65(12), 3691-3704 (2016). 